\title{
Overview of Post-Stroke Epilepsy
}

\author{
Wadi Bnouhanna $^{*}$, Amal Satté2 , Jamal Mounach ${ }^{2}$, Hamid Ouhabi ${ }^{2}$ \\ ${ }^{1}$ Department of Neurology A and Neuropsychology, University Hospital IbnSina, Rabat, Morocco \\ ${ }^{2}$ Department of Neurophysiology, Military Hospital Mohammed V, Rabat, Morocco \\ Email: *w.bnouhanna@gmail.com
}

How to cite this paper: Bnouhanna, W., Satté, A., Mounach, J. and Ouhabi, H. (2019) Overview of Post-Stroke Epilepsy. Open Access Library Journal, 6: e5705. https://doi.org/10.4236/oalib.1105705

Received: August 14, 2019

Accepted: August 30, 2019

Published: September 3, 2019

Copyright $\odot 2019$ by author(s) and Open Access Library Inc.

This work is licensed under the Creative Commons Attribution International License (CC BY 4.0).

http://creativecommons.org/licenses/by/4.0/

\begin{abstract}
Stroke is the most common cause of seizures, symptomatic epilepsy, and status epilepticus in elderly. Symptomatic stroke-related seizures symptoms are dependent on lesions topography. All types of focal seizures can be observed. Many clinical studies make a distinction between early (within 7 to 14 days of stroke onset) and late (beyond 14 days of stroke onset) seizures based on presumed physiopathological differences. The diagnosis of a seizure during the acute phase of a stroke can be difficult in the absence of a witness when there are disorders of vigilance and speech. The use of antiepileptic drugs is discussed in all cases. New antiepileptic drugs appear to be most appropriate. The aim of this study is to present an overview of the epidemiological, clinical and therapeutic features of vascular epilepsy based on meta-analyses and the more recently published expert recommendations.
\end{abstract}

\section{Subject Areas}

Geriatrics

\section{Keywords}

Post-Stroke Epilepsy, Vascular Epilepsy, Stroke, Antiepileptic Drugs

\section{Introduction}

Stroke is the most common cause of seizures, symptomatic epilepsy, and status epilepticus (SE) in the elderly; they all can present clinical feature of acute stroke. While the incidence of epilepsy rises significantly after 60 years, it can reach 139 per 100,000 in people over the age of 75 . The leading cause of epilepsy or seizures within this population is stroke. Recent data suggest that the occurrence of a first unprovoked seizure can be an independent risk factor for subsequent stroke. The relationship between stroke, seizures, and epilepsy raises many questions on risk factors, possible prevention, physiopathology, therapeutic in- 
dications, treatment effects, recovery capabilities after stroke, and comorbidity in specific populations such as the elderly and infants. The aim of this study is to present an update of the epidemiological, clinical and therapeutic features of vascular epilepsy.

\section{Methods}

A non-exhaustive literature search using the keywords "vascular epilepsy" and "post-stroke epilepsy" was conducted on PubMed and Science Direct, including articles appeared after the year 2000, in English and in French. The main objective of this review is not to claim a detailed and comprehensive analysis of the issue, but rather to present a global view on this disease, its frequency, clinical diagnostic and different therapeutics what we consider most relevant to clinical practice.

\section{Epidemiology}

Cerebrovascular lesions are responsible for over $10 \%$ of seizures in adults and $50 \%$ of epilepsy of the elderly. In recent studies, the risk of post-stroke epilepsy is multiplied by 20 compared with the risk in the general population of the same age group [1]. In the Netherlands, 41,000 patients suffer from a stroke annually. Late epileptic seizures develop in $2.5 \%-15 \%$ of stroke patients. Recurrent late symptomatic epileptic seizures develop $48 \%$ - $89 \%$ of stroke patients with a remote seizure. However, the prevalence of post-stroke seizures is quite low. It varies from $5 \%$ to $20 \%$ (average $10 \%$ ) in the most recent study [2]. This wide variation is probably due to heterogeneous study designs, inconsistent terminology, and ambiguities in the identification of seizures and epilepsy. 1 of 6 children suffering from stroke had a remote seizure by 5 years post-stroke, which increases to 1 of 3 children by 10 years post-stroke. Prior pediatric stroke studies have reported that $7 \%$ to $29 \%$ of children presented remote seizures or epilepsy. A recent study of prenatal and infantile intracerebral hemorrhage states that $13 \%$ of children developed epilepsy after 2 years [3]. In the elderly aged above 60 , post-stroke epilepsy represents more than $45 \%$ of all incident cases.

Most studies differentiate early seizures and late seizures. Early seizures are symptomatic attacks of acute brain disorder and are not synonymous of epileptic disease. It is usually a single seizure, and the majority of them occur during the first 24 hours. Their frequency varies from $2 \%$ to $8 \%$ in recent prospective studies. These variations are partly caused by the different understanding of the term "early", ranging from 24 - 48 hours to one month after the stroke in different studies [4].

The criteria proposed by the International League Against Epilepsy are modeled on studies that define the post-traumatic seizures as the period of occurrence of early seizures in the first seven days after the stroke [5]. Even though this distinction may seem arbitrary, it is crucial since the two types of seizures, early and late, presumably correspond to different pathophysiological mechanisms. The incidence of late seizures can reach $67 \%$ of cases within two years fol- 
lowing stroke [4]. However, the risk is higher between the sixth and twelfth months. The frequency of recurrent post-stroke seizures is lower. The risk is estimated between $1 \%$ and $5 \%$ within the first year after stroke, and approximately $5 \%$ in five years. However, it differs depending on the type and the location of the stroke [5] [6].

\section{Physiopathology}

The distinction between seizures occurring during the acute phase of stroke and those occurring late (usually beyond 2 weeks) falls within important physiopathological and therapeutic considerations. Seizures or status epilepticus in the initial period can be considered "occasional" in the context of physiological brain response to acute attack. The Physiopathology of post-stroke seizures is not completely understood. Hypothesis has been proposed, distinguishing between late crises and early seizures. During the acute phase of infarction, there are two main phenomenon involved in the genesis of an epileptic seizure:

- Significant alterations in calcium homeostatic mechanisms occur in association with stroke-induced epilepsy and suggest that these changes may play a role in both induction and maintenance of epileptic phenotype in this model [7] [8].

- Tissue hypoxia secondary to the fall of cerebral blood flow, liberation of excitotoxic amino acids such as glutamate, and intracellular accumulation of calcium and sodium cause neuronal depolarization leading to seizure. Repetitive neuronal discharges could occur within networks of surviving neurons exposed to glutamate. A decrease in the efficiency of gamma-aminobutyric acid (GABA) inhibition causing cortical hyperexcitability through N-methyl-D-aspartate (NMDA) receptors is suggested [1] [5].

The ischemic penumbra may also play a part intriggering the seizures. The low frequency of early seizures suggests that other factors than ischemia are involved mainly local factors (reperfusion damage caused by early recanalization) and general factors (hyperglycemia, medication weaning, electrolyte disorders, and hypoxia secondary to pulmonary disease). The role of the mass effect, ischemia, and degradation products of hemoglobin is suggested in genesis of seizures secondary to hemorrhagic stroke.

Late seizures are linked to an "epileptogenic organization" of stroke scar area due to structural modifications. Experimental studies suggest that the modifications of neuronal membranes properties and the pathological neurotransmitter balance and organization of connections make the neural networks abnormally excitable and hypersynchronized. The epileptogenic role of iron deposits is the most commonly accepted hypothesis to explain the high prevalence of post-hemorrhagic stroke seizures [8].

\section{Risk Factors}

Several studies have shown that the frequency of early seizures is higher in case 
of cerebral hemorrhage ( $3 \%$ to $19 \%$ ), cerebral venous thrombosis (CVT) (12\% to $34 \%$ ), or subarachnoid hemorrhage (SAH) (4\% to $18 \%$ ) than in ischemic stroke (2\% through 6\%) [1] [5] [6]. The cortical topography of stroke is one of the most relevant predictor factors of early seizures. Seizures have sometimes been described in cases of deep infarcts [9]. The risk of early seizures is higher in case of lobar hemorrhage than in deep hemorrhage [10]. The risk factors of seizures in TVC are focal deficits, thrombosis of cortical veins, and brain parenchymalesion [10]. Cortical infarcts were identified as a risk factor for late seizures or epilepsy. Other risks factors of seizures after stroke have been reported: severity of stroke, infarct size, multiple infarcts, and existence of hemorrhagic transformation. Few studies have reported a higher frequency of early seizures in cardioembolic mechanism [1] [5] [11].

In intracranial haemorrhage, the size of the haemorrhage and the amount of blood in the subarachnoid space and ventricles do not seem to be predictive of seizure. A retrospective analysis investigated the predictive factors of early and late onset seizures after evacuation of intracerebral hemorrhage (ICH), in an attempt to guide antiepileptic drug management in this population. It was concluded first that the presence of subdural hematoma and increased INR are predictive of late-onset seizures in patients undergoing clot evacuation after ICH and that long-term use of antiepileptic therapy should be further studied in patients with these radiographic and clinical characteristics [10]. The occurrence of early seizure seems to be associated with an increasing risk of late seizure. Other risk factors for late seizure are identified: initial severity of infarction, stroke size, disability, stroke recurrence, and preexisting dementia.

In pediatric population, there are few studies about post-stroke seizures considering the rarity of stroke in children. Seizures can be a sign of acute stroke or can occur at distance [12]. A risk factor of early seizures is cortical location of stroke as in adults. Acute seizures at the time of stroke are the major risk factor for predicting active epilepsy. However, some specific epilepsy syndromes of the child could be related to the occurrence of subcortical stroke, including the thalamus which was recently reported for the non-idiopathic syndrome of continuous spikes and waves during sleep (CSWS) [5] [13]. A German team evaluated the association of post-stroke seizures and conventional vascular risk factor through a cohort study including 593 consecutive patients with different types of cerebrovascular events [14]. They concluded that epileptic seizures occur particularly after major strokes and insinus thrombosis. However, conventional vascular risk factors were not associated with the occurrence of post-stroke seizures.

In order to predict post-stroke epilepsy and to choose the best point in time for anticonvulsant treatment, a prospective study evaluated 264 consecutive stroke patients and assessed their post-stroke epilepsy risk within a follow-up of 1 year. Data on ten risk items concerning the stroke location, neurological deficit persistence, stroke subtype, established diagnosis of vascular encephalopathy, and early and late-onset seizures were collected using a post-stroke epilepsy risk 
scale (PoSERS). The total scale showed moderate sensitivity (70\%) and positive predictive value $(87.5 \%)$ while specificity $(99.6 \%)$ and negative predictive value (98.8\%) were relatively high. The EEG showed little value in predicting post stroke epilepsy. According to the authors, PoSERS appears to be a valuable tool to predict the risk for post-stroke epilepsy within the first few days after a stroke [14].

\section{Clinical Features}

Symptomatic strokes seizures symptoms are dependent on lesions topography. All types of focal seizures can be observed. Semiological analysis of seizures is limited by the presence of more or less important focal deficits, vigilance disorders, language disorders and the retrospective character of some studies. The most frequent early seizures are either focal simple or complex. They may be secondarily generalized or generalized with unknown focal onset [14] [15].

Stroke is the leading cause of status epilepticus in adults, varying between $22 \%$ and $32 \%$ of cases of status epilepticus seizures occurring in adults. During stroke, status epilepticus occurs in $1 \%$ to $1.4 \%$ of patients. Within this population with stroke, the occurrence of status epilepticus was from $2.8 \%$ to over $10 \%$. Status epilepticus occurring at early phase of stroke is associated to a highmortality, with approximately $50 \%$ of fatal outcomes [16] [17].

\section{Diagnosis}

The diagnosis of a seizure during the acute phase of a stroke can be difficult in the absence of a witness when there are disorders of vigilance and speech. Focal seizures are usually quite easy to differentiate from transient ischemic attack (TIA) during which the clinical events are a neurological deficit that is longer (several minutes) than that of an epileptic seizures (seconds to a few minutes). Conversely, focal seizures with purely deficit signs were reported with short episodes, recurrent aphasia, and sensory or motor deficit. The stereotyped nature of the episodes, their short duration, the identification of contralateral paroxysmal abnormalities in the EEG, and the efficiency of antiepileptic drugs makes it possible to differentiate it from TIA [5] [16]. Limb shaking, which is a non-epileptic paroxysmal motor phenomenon associated with lower flow in the contralateral carotid territory, may be more misleading. Limb shaking is a repeated and rhythmic involuntary movement that is caused by contralateral carotid stenosis. The absence of consciousness disorders and walking seizures, the link between the appearance of signs in the upright posture and their disappearance while lying down, the normality of the EEG during symptoms, and the inefficiency of antiepileptic treatment confirm the diagnosis.

EEG is often abnormal in acute stroke. The most common abnormalities are focal slowing activity. It can be either a rhythmic slow wave or periodic lateralized epileptiform discharges (PLED). These EEG abnormalities are not specific to seizures. The positive predictive value of these paroxysmal abnormalities on 
the occurrence of post-stroke seizure is considered low by most authors. The existence of paroxysmal EEG abnormalities after stroke is not an argument to suggest a preventive antiepileptic treatment. However, the EEG is essential for non-convulsive status epilepticus, and the diagnosis must be realized in emergency in case of disorders of consciousness or unexplained confusional state [1] [5] [16].

\section{Treatment}

The role of antiepileptic treatment in post-stroke seizures remains poorly defined. In the absence of randomized therapeutic trials sufficiently large, most therapeutic indications are based on observational studies and professional consensus. The administration of a preventive anti-epileptic treatment in cerebral infarction is not currently recommended in acute phase. For cerebral hemorrhage, although there is no scientific evidence, the U.S. guidelines consider the possibility of a brief preventive anti-epileptic treatment in the acute phase of lobar hemorrhage. In a post-stroke late seizure, the administration of treatment is recommended for prevention of remote seizures for both cerebral infarction and hemorrhage. Contributing factors (drug withdrawal, metabolic disturbances, hypoxia ...) should be investigated and treated. Large randomized studies have shown that if the antiepileptic treatment is effective in the prevention of epileptic seizures in the acute phase of brain injury, it does not decrease either mortality or the risk of subsequent epilepsy. There is also no favorable impact on functional outcomes. Based on these results, it does not seem to be beneficial to prolong AED therapy for early seizure post-stroke. In contrast, it is consistent to consider that the occurrence of a seizure beyond the early period of stroke (the first 2 weeks) expose to a higher risk of recurrence which justifies prescribing long term antiepileptic treatment [1] [5].

It has been demonstrated that people with epilepsy have a poor health status and are more likely to report poor health related quality of life than the general population. Even though seizure freedom should be a primary clinical goal, patients with epilepsy often have comorbidities. Thus, hepatic and renal function, drug interactions especially with anticoagulants, and the high susceptibility of this population to treatment side effects should always be considered. In this respect, it is believed that the comorbidities of patients with epilepsy should be taken into account when deciding about an antiepileptic drug (AED) and it should improve the overall health and quality of life of patients with epilepsy [18].

Until recently, few options were available for the pharmacological treatment of epilepsy. In the last few years, however, more than ten new AEDs have been used. Although these new AEDs have not demonstrated greater efficacy than older drugs, many of them have an improved pharmacokinetic profile and a better tolerance. These advantages make these AEDs potentially useful in patients with epilepsy and other comorbidities [19] [20] [21]. A Spanish team has 
conducted an analysis of scientific literature about the indication of antiepileptic drug according to comorbidities factor and taking into account the major comorbidities associated with stroke, which are cardiovascular disease, kidney disease, and mental disability. Based on this review two antiepileptics are distinguished leviteacetam and lamotrigine [18]. It is believed that lamotrigine is less convenient given that the titration period is too long to control lesional epilepsy with a high potential for recurrence. While levetiracetam exhibits favorable characteristics: safety, low potential for interaction, no active metabolites, short elimination half-life, no detrimental effects on sleep architecture, and no major negative effects on cognition and efficiency profiles. These characteristics make it suitable for use in the elderly population [22]. An Italian team evaluated the efficacy and safety of intravenously administered lacosamide (iv LCM) in post-stroke non convulsive status epilepticus (NCSE) in elderly patients. They suggested that LCM exhibits safety and efficacy profiles, which make it an optimal candidate as a first-choice drug against post-stroke NCSE in elderly patients [8] [23].

Statin therapy has been shown to be beneficial to prevent post-stroke seizures in the acute phase. The positive effect of statins results from their anticonvulsant/inflammatory mechanism in preventing injury to the blood-brain barrier [8]. In the light of all these elements, four clinical situations are distinguished [21]:

1) Early seizure with contributing factors: no antiepileptic treatment.

2) Early seizure with limited stroke and minor disability: antiepileptic treatment is considered case by case during limited time.

3) Early seizure with extended stroke and severe disability, in cases of cerebral venous thrombosis or lobar hemorrhage: antiepileptic treatment is justified but limited in time.

4) Late seizure: AED therapy is recommended for a long period.

\section{Prognosis}

The impact of post-stroke seizures on mortality and functional outcome remains debated. In theory, the occurrence of seizures in the acute phase of stroke may aggravate metabolic dysfunctions with their deleterious effect on brain tissue. Several studies have reported increased mortality in early seizures after stroke. Recurrence of seizures is often infrequent, and post-stroke epilepsy drug resistant appears to be rare. The existence of post-stroke epilepsy is likely to decrease the quality of life of patients often impaired by residual disability, but its real impact has not been specifically studied [1] [24] [25].

The immediate prognosis of stroke status epilepticus is quite dark with high mortality ranging from $30 \%$ to $53 \%$ with an increase of neurological deficit that might be irreversible. The stroke status epilepticus recurrences are not uncommon, especially when they are early. However, the occurrence of stroke status epilepticus in early period of stroke does not seem to increase the risk of subse- 
quent epilepsy [5].

\section{Conclusion}

About $5 \%$ of patients will present one or more seizures in the acute phase or at the distance of stroke. This risk is higher in hemorrhagic stroke, extended ischemic stroke with severe neurological disability, cortical topography, and early seizure orvenous infarction. The use of antiepileptic drugs is discussed in all cases. New antiepileptic drugs appear to be most appropriate, mainly levetiracetam.

\section{Conflicts of Interest}

The authors declare no conflicts of interest regarding the publication of this paper.

\section{References}

[1] Vercueil, L. (2007) Seizures, Epilepsy and Stroke. La Presse Médicale, 36, 176-181. https://doi.org/10.1016/j.lpm.2006.11.001

[2] Van Tuijl, J., van Raaka, E.P.M., de Kroma, M.C.T.F.M., Loddera, J. and Aldenkamp, A.P. (2011) Early Treatment after Stroke for the Prevention of Late Epileptic Seizures: A Report on the Problems Performing a Randomised Placebo-Controlled Double-Blind Trial Aimed at Anti-Epileptogenesis. Seizure, 20, 285-291. https://doi.org/10.1016/j.seizure.2010.12.012

[3] Fox, C., et al. (2013) Acute Seizures Predict Epilepsy after Childhood Stroke. Annals of Neurology, 74, 249-256. https://doi.org/10.1002/ana.23916

[4] Xu, M.Y. (2019) Poststroke Seizure: Optimising Its Management. Stroke and Vascular Neurology, 4. https://doi.org/10.1136/svn-2018-000175

[5] Lamy, C. (2008) Epilepsy and Stroke. Revue Neurologique, 164, 841-845. https://doi.org/10.1016/j.neurol.2008.07.006

[6] Chen, T.C., Chen, Y., Cheng, P. and Lai, C. (2012) The Incidence Rate of Post-Stroke Epilepsy: A 5-Year Follow-Up Study in Taiwan. Epilepsy Research, 102, 188-194. https://doi.org/10.1016/j.eplepsyres.2012.06.003

[7] Sun, D., Sombati, S., Blair, R. and DeLorenzo, R. (2004) Long-Lasting Alterations in Neuronal Calcium Homeostasis in an in Vitro Model of Stroke-Induced Epilepsy. Cell Calcium, 35, 155-163. https://doi.org/10.1016/j.ceca.2003.09.003

[8] Feyissa, A.M., Hasan, T.F. and Meshia, J.F. (2019) Stroke-Related Epilepsy. European Journal of Neurology, 26, 18-e3. https://doi.org/10.1111/ene.13813

[9] De Reuck, J., Nagya, E. and Van Maele, G. (2007) Seizures and Epilepsy in Patients with Lacunar Strokes. Journal of the Neurological Sciences, 263, 75-78.

https://doi.org/10.1016/j.jns.2007.06.004

[10] Garrett, M., et al. (2009) Predictors of Seizure Onset after Intracerebral Hemorrhage and the Role of Long-Term Antiepileptic Therapy. Journal of Critical Care, 24, 335-339. https://doi.org/10.1016/j.jcrc.2008.10.015

[11] Kammersgaard Lars, P. and Olsen, T.S. (2005) Post Stroke Epilepsy in the Copenhagen Stroke Study: Incidence and Predictors. Journal of Stroke and Cerebrovascular Diseases, 14, 210-214. https://doi.org/10.1016/j.jstrokecerebrovasdis.2005.07.001

[12] Strzelczyk, A., Haag, A., Raupach, H., Herrendorf, G., Hamer, H.M. and Rosenow, 
F. (2010) Prospective Evaluation of a Post-Stroke Epilepsy Risk Scale. Journal of Neurology, 257, 1322-1326. https://doi.org/10.1007/s00415-010-5520-9

[13] Leone, M., et al. (2009) Risk Factors for a First Epileptic Seizure after Stroke: A Case Control Study. Journal of the Neurological Sciences, 277, 138-142. https://doi.org/10.1016/j.jns.2008.11.004

[14] Conrad, J., Pawlowski, M., Dogan, M., Kovac, S. Ritter, M.A. and Evers, S. (2013) Seizures after Cerebrovascular Events: Risk Factors and Clinical Features. Seizure, 22, 275-282. https://doi.org/10.1016/j.seizure.2013.01.014

[15] Chun-Ming, C., Tsoi, T.-H., Au-Yeung, M. and Suk-Yan Tang, A. (2003) Epileptic Seizure after Stroke in Chinese Patients. Journal of Neurology, 250, 839-843. https://doi.org/10.1007/s00415-003-1091-3

[16] Okuda, S., Takano, S., Ueno, M., Hamaguchi, H. and Kanda, F. (2012) Clinical Features of Late-onset Poststroke Seizures. Journal of Stroke and Cerebrovascular Diseases, 21, 583-586. https://doi.org/10.1016/j.jstrokecerebrovasdis.2011.01.006

[17] DeReuck, J., De Groote, L. and Van Maele, G. (2008) Single Seizure and Epilepsy in Patients with a Cerebral Territorial Infarct. Journal of Neuroscience, 271, 127-130. https://doi.org/10.1016/j.jns.2008.04.005

[18] Ruiz-Gimenez, J., Sánchez-Álvarez, J.C., Cañadillas-Hidalgo, F. and Serrano-Castro, P.J. (2010) Antiepileptic Treatment in Patients with Epilepsy and Other Comorbidities. Seizure, 19, 375-382. https://doi.org/10.1016/j.seizure.2010.05.008

[19] Belcastro, V., et al. (2008) Levetiracetam in Newly Diagnosed Late-Onset Post-Stroke Seizures: A Prospective Observational Study. Epilepsy Research, 82, 223-226. https://doi.org/10.1016/j.eplepsyres.2008.08.008

[20] Belcastro, V., Pierguidi, L. and Tambasco, N. (2011) Levetiracetam in Brain Ischemia: Clinical Implications in Neuroprotection and Prevention of Post-Stroke Epilepsy. Brain \& Development, 33, 289-293. https://doi.org/10.1016/j.braindev.2010.06.008

[21] Holtkamp, M., et al. (2017) European Stroke Organisation Guidelines for the Management of Post-Stroke Seizures and Epilepsy. European Stroke Journal, 2, 103-115.

[22] Werhahn, K., Klimpe, S., Balkaya, S., Trinka, E. and Krämer, G. (2011) The Safety and Efficacy of Add-on Levetiracetam in Elderly Patients with Focal Epilepsy: A One-Year Observational Study. Seizure, 20, 305-311. https://doi.org/10.1016/j.seizure.2010.12.015

[23] Belcastro, V., et al. (2013) Intravenous Lacosamide as Treatment Option in Post-Stroke Non Convulsive Status Epilepticus in the Elderly: A Proof-of-Concept, Observational Study. Seizure, 22, 905-907. https://doi.org/10.1016/j.seizure.2013.07.011

[24] Chang, C.S., et al. (2014) Patients with Epilepsy Are at an Increased Risk of Subsequent Stroke: A Population-Based Cohort Study. Seizure, 23, 377-381. https://doi.org/10.1016/j.seizure.2014.02.007

[25] Hamidou, B., et al. (2013) Prognostic Value of Early Epileptic Seizures on Mortality and Functional Disability in Acute Stroke: The Dijon Stroke Registry (1985-2010). Journal of Neurology, 260, 1043-1051. https://doi.org/10.1007/s00415-012-6756-3 